\title{
ON THE CHANGE IN RADAR CROSS-SECTION OF A SPHERICAL SATELLITE CAUSED BY A PLASMA SHEATH
}

C. L. DOLPH and H. WEH

The Radiation Laboratory of The University of Michigan, Ann Arbor, Michigan

\begin{abstract}
A uniform neutral dilute ionized gas is assumed to be perturbed by a sphere moving through it. The radar return from the disturbed region is obtained by integrating the Compton scattering from the electrons, taking phase into account, but ignoring secondary scattering and attenuation. The electron density distribution for this computation is obtained by integration of the zeroth order velocity distribution function for neutral particles obtained by Wang Chang( ${ }^{(\theta)}$ as a solution of the Boltzmann transport equation.

Numerical results are obtained for the perturbation of the electron distribution by a sphere travelling at $8 \mathrm{~km} / \mathrm{sec}$ and an altitude of $500 \mathrm{~km}$, and for the radar cross-section of this perturbed region when viewed broadside.*
\end{abstract}

\section{INTRODUCTION}

A sphere is assumed to move with a constant velocity, $V$, through a dilute, electrically neutral, ionized gas that, in its unperturbed state, is assumed to have a uniform number distribution of electrons, $n$. The sphere disturbs the distribution of electrons to a non-uniform one, $N$, with an excess ahead of the sphere and a deficiency behind it. The simplest estimate of the effect of this non-uniform, but everywhere dilute distribution on the radar return is obtained by summing the scattering by the individual electrons accelerated by the incident field. Electrons only are considered since their return is far greater than that of the much heavier positive ions or the Rayleigh scattering from non-ionized particles. The incident field on each electron is assumed to be a plane wave; this implies that secondary scattering is ignored. The approach is thus directly analogous to that used to determine the radar return from underdense meteor trails. (1)

There are three parts to the paper. The first part consists of a formulation of the expression for the back-scattered energy. This expression involves the perturbed density distribution. The condition of neutrality is used in determining this distribution since it forces the electron motion to be governed by the positive ions whose velocities and mass are similar to that of the neutral particles. This will be discussed in the second part of the paper where expressions for the distribution are found. In the third part numerical results for a specific sphere velocity and altitude are presented.

\section{THE SCATTERING INTEGRAL}

The radiation field of each electron yields a back-scattered power per unit solid angle per electron for unit incident power density given by

$$
\sigma_{e}=\left[e^{2} /\left(4 \pi \varepsilon_{0} m c^{2}\right)\right]^{2} \text {. }
$$

Here $e$ is the charge on the electron, $m$ its mass, $\varepsilon_{0}$ the permittivity of free space, $c$ the velocity of light and m.k.s. units are to be used. The incident power is given by $P G /\left(4 \pi r^{2}\right)$ where $P$ is the total power emitted from the radar antenna, $G$ the antenna gain and $r$ the distance from antenna to electron. The effective collecting area of the antenna is $G \lambda^{2} /(4 \pi)$ so that the

* The theoretical work and preliminary computations were supported by USAF Contract AF 30(602)-1853. The final machine computations were carried out as an unsponsored faculty research project MO2-N at the Computation Laboratory of The University of Michigan. 
scattered power per electron received by the radar for large $r$ is

$$
S_{e}=\frac{P G^{2} \lambda^{2} \sigma_{e}}{16 \pi^{2} r^{4}}
$$

We now assume the radar is well out of the ionized region of interest so that $r$ is always large. Then the net power received from this region is

$$
S=\frac{\lambda^{2} \sigma_{e} P}{16 \pi^{2}}\left|\int \frac{G}{r^{2}} e^{2 i k r} N d v\right|^{2}
$$

where $d v$ is a volume element. For simplicity a beam width wide enough to be essentially constant over the disturbance is assumed and the slowly varying factor $r^{-2}$ replaced by the range $R_{0}$ to the sphere and removed from under the integral sign. Finally, $N$ is referred to the constant $n$ by writing $N=(N-n)+n$. The integral of $n$ exp ( $2 i k r)$ vanishes except for contributions at the "edges" of the region of integration. Of course the distribution $n$ extends beyond the beam-width of the radar and thus we know these "edges" are not physically significant. They may be neglected with the result that the desired quantity, the net power received due to the disturbance of the density distribution is given by

$$
S_{D} \sim \frac{\lambda^{2} \sigma_{e} P G^{2}}{16 \pi^{2} R_{0}^{4}}\left|\int e^{2 i k r}(N-n) d v\right|^{2} .
$$

The integration is to be extended over the region of interest. In general this will include the entire region over which $N-n$ differs appreciably from zero. However, one might also be interested in considering separately the effects of the region ahead of the sphere and the region behind it. If these were to act as independent scatterers the average returned power (averaged over all relative phases) would be the sum of two expressions $S_{D_{1}}$ and $S_{D_{2}}$ corresponding to $S_{D}$ with the integration in $S_{D_{1}}$ over the region ahead of the sphere and the region in $S_{D_{2}}$ over the region behind the sphere.

To put the integral in $S$ in a form suitable for computation it is convenient to refer to Fig. 1.

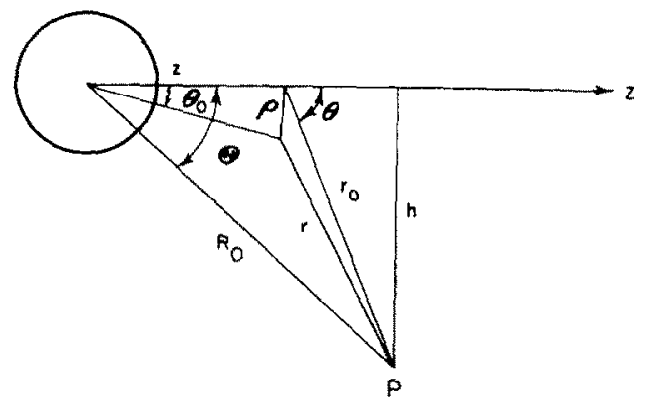

Fig. 1. Satellite-receiver geometry.

The density $N$ must be symmetric about $z$ so that it is convenient to use cylindrical coordinates $\rho, \psi, z$ in the integration. Furthermore, one can simplify the integral by using in the expression for $\mathbf{r}(\boldsymbol{\rho})$

$$
(\mathbf{r} \rho)=\mathbf{r}_{0}-\rho ; \quad r^{2}=r_{0}^{2}+\rho^{2}-2 \mathbf{r} \cdot \boldsymbol{\rho}
$$

the fact that $\frac{\rho}{r_{0}} \ll 1$, so that

$$
r=r_{0}\left[1+\frac{\rho^{2}}{r_{0}^{2}}-\frac{2 \rho}{r_{0}} \sin \theta \cos (\phi-\psi)\right]^{1 / 2}
$$

$$
\begin{aligned}
& \sim r_{0}-\rho \sin \theta \cos (\phi-\psi)+\frac{1}{2} \frac{\rho^{2}}{r_{0}}- \\
& \quad \frac{1 \rho^{2}}{8 r_{0}} \sin ^{2} \theta \cos ^{2}(\phi-\psi)+\ldots
\end{aligned}
$$

In turn $r_{0}$ is approximated by

$r_{0} \simeq R_{0}+\frac{z^{2}}{2 R_{0}}-2 z \cos \Theta-\frac{1}{8} \frac{z^{2}}{R_{0}} \cos ^{2} \Theta+\ldots$

We shall be interested in $60^{\circ}<\Theta<120^{\circ}$ and hence will neglect the last term as well as those of higher order in $z / R_{0}$. Then

$$
\begin{aligned}
S=\frac{\lambda^{2} \sigma_{e} P G^{2}}{16 \pi^{2} R_{0}^{4}} & \int_{0}^{2 \pi} d \psi \int_{-\infty}^{\infty} d z \int_{0}^{\infty} \\
& \left.\rho d \rho[N(\rho, z)-n] e^{2 i k r(\rho, \psi, z)}\right|^{2}
\end{aligned}
$$

$\simeq \frac{\lambda^{2} \sigma_{e} P G^{2}}{4 R_{0}^{4}} \mid \int_{-\infty}^{\infty} d z \cdot \exp \left[2 i k\left(\frac{z^{2}}{2 R_{0}}-z \cos \theta\right)\right]$ 


$$
\left.\left\{\int_{0}^{\infty} \rho d \rho[N(\rho, z)-n] J_{0}[2 k \rho \sin \theta(z)]\right\}\right|^{2}
$$

The term $\frac{1}{2} \rho^{2} / r_{0}$ is neglected in the phase since it will appreciably affect the phase only if $\Theta=90^{\circ}$ and then only where $\rho$ exceeds $\sim 0.1 \sqrt{ } R_{0}$. For such large $\rho$ 's the amplitude $N-n$ is negligible. This is not quite true for corresponding values of $z$. In this consideration we have assumed $k<\sim 20 \mathrm{~m}^{-1}$. Note that in this integral $\sin \theta(z)=R_{0} \sin \Theta / r_{0}(z)$.

\section{COMPUTATION OF THE ELECTRON DISTRIBUTION}

The steady-state problem of a point charge moving through a fully ionized medium of sufficiently low density has been treated by Kraus and Watson. ${ }^{(2)}$ Their work was extended to the case where a constant magnetic field is present by Greifinger. ${ }^{(3)}$ A good deal of insight into the physical meaning of the above theories which started from the linearized LandauVaslov equation was provided by the report of Pappert. ${ }^{(4)}$ In this report Pappert deduced the results of Kraus and Watson from the random phase approximation of Bohm and Pines ${ }^{(5)}$ and also demonstrated the equivalence of these methods to the linearization of the equations of motion and continuity for the ions and electrons under the assumption that an isothermal state exists.

The problem of an object of finite size has been approached by using the expression obtained in 1950 by Wang Chang. ${ }^{(6)}$ She obtained the zeroth order velocity distribution function for a sphere of radius $R$ at rest in a neutral gas with a streaming velocity $V$ under the assumption that the sphere was sufficiently small and the gas sufficiently dilute that the collisions between the main stream particles and those reflected from the sphere could be neglected. The distribution function so determined satisfies:

(a) The collision-free Boltzmann transport equation in the absence of external forces;

(b) A boundary condition on the surface of the sphere that implies that the sphere neither absorbs nor emits gas particles by itself so that all particles that hit the sphere are re-emitted;

(c) The property that it reduces to the Maxwell-Boltzmann distribution around the steaming velocity at infinity independent of angle.

In addition, this distribution allows for arbitrary amounts of diffuse or specular reflection at the spherical surface. Since the region of interest here involves velocities of the sphere much greater than that of the ions and at the same time much smaller than that of the electrons and altitudes where the mean free paths are large compared to the expected dimensions of the disturbed area, Chang's distribution may be used to provide an order of magnitude estimate of the electron distribution around the sphere when it is assumed that the charge on the sphere is so small that it can be ignored so that the ions will (to all intents and purposes) behave as neutral particles as far as their interaction with the sphere is concerned. The strong Coulomb forces should provide electrical neutrality which will then force the electrons to assume a distribution identical in form to Chang's in which only the mass and velocity parameters can be different.

This use of electrical neutrality, while appropriate in ionospheric physics, is less exact than the assumptions usually used in physics of confined plasmas where ${ }^{(7)}$ it is more customary

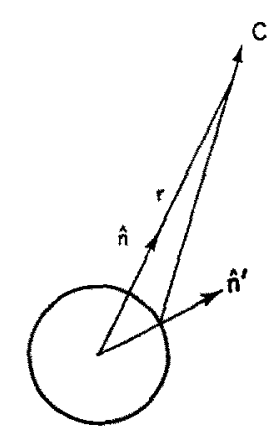

Fig. 2. Relation between $\mathbf{C}$ and $\hat{\mathbf{n}}$. 
to assume electrical neutrality except for consideration of Poisson's equation while here we ignore any deviations from neutrality in this equation as well. While it would be more exact to assume the Chang distribution as the first term in a perturbation procedure for the LandauVlasov equations, it is unlikely that such a refined analysis would affect the radar crosssection results.* Other approaches such as that used by Bernstein and Rabinowitz ${ }^{(9)}$ in their discussion of spherical probes would seem to encounter even more intractable analytical difficulties were the Chang distribution to be used in place of the mono-energetic one used by them.

The zeroth order velocity distribution obtained by Chang is expressed in terms of the following variables.

$\mathbf{V}^{\prime}=$ the velocity of the main stream

$R=$ the radius of the sphere

$r=$ the point under consideration, or the point at which the velocity is being calculated

$\hat{n}=$ the outward normal to the sphere which passes through the point; $\mathbf{r}=r \hat{\mathbf{n}}$ if the centre of the spherc is taken as the origin of the co-ordinate system

$\mathbf{C}^{\prime}=$ the velocity vector of gas particles

$\alpha=$ the fraction of molecules that is diffusely reflected from the sphere

$1-\alpha=$ the fraction of molecules that is specularly reflected from the sphere

$\mathrm{V}=\mathbf{V}^{\prime} / \sqrt{2 k t / m}$, non-dimensional streaming velocity

$\mathbf{C}=\mathbf{C}^{\prime} / \sqrt{2 k t / m}$, non-dimensional velocity vector

* Actually the indicated procedure for the second approximation is presently under investigation under another contract for a different purpose. Preliminary analysis seems to indicate the existence of oscillatory solutions for the electron density with frequencies of the order of those characteristic for plasmas modified by an increment dependent upon electron temperature and form factors appropriate to the geometry. See Ref. (8). $\hat{n}^{\prime}=$ the normal to the sphere at the point from which the molecules arriving at $\mathbf{r}$ with velocity $\mathbf{C}$ originated. From Fig. 2 it is found that

$$
\hat{n}^{\prime} \quad \stackrel{\mathbf{r}}{\mathrm{r}}-\frac{\mathbf{r} \cdot \mathrm{C}}{R C^{2}} \mathrm{C}+r \sqrt{\frac{(\mathrm{r} \cdot \mathrm{C})^{2}}{r^{2} C^{2}}-\left(1-\frac{R^{2}}{r^{2}}\right)} \frac{\mathrm{C}}{R C}
$$

$\hat{n}_{C}=$ unit vector in the direction of $\mathbf{C}$

$n=$ the number of particles present in the unperturbed state

$\operatorname{erfc}(x)=\frac{2}{\sqrt{\pi}} \int_{x}^{\infty} \exp \left(-t^{2}\right) d t=1-\operatorname{erf}(x)$

$S=S\left(x, y, z, \hat{n}_{c}\right)$, a discontinuous function which is zero if the particles of velocity $C$ at point $\mathbf{r}(y, x, z)$ come from the sphere and which is one otherwise. That is, $S=0$ if $\hat{n}_{C}$ points away from the sphere and lies in the solid angle subtended by the sphere at the point under consideration.

The distribution can be written as

$$
\begin{aligned}
& f(\alpha)=\frac{n}{\pi^{3 / 2}}\left\{S \exp -(\mathbf{C}-\mathbf{V})^{2}+\alpha\left[\exp -\left(\mathbf{V} \cdot \hat{n}^{\prime}\right)^{2}-\right.\right. \\
& \left.-\sqrt{\pi}\left(\mathbf{V} \cdot \hat{n}^{\prime}\right) \operatorname{erfc}\left(\mathbf{V} \cdot \hat{n}^{\prime}\right)\right](1-S) \exp -C^{2}+ \\
& \left.+(1-\alpha)(1-S) \exp -\left[\mathbf{C}-\mathrm{V}-2 \hat{n}^{\prime}\left(\hat{n}^{\prime} \cdot C\right)\right]^{2}\right\}
\end{aligned}
$$

The function $S$ which is described above can be represented analytically as

$$
\begin{aligned}
S=\frac{1}{2}\left(1-\operatorname{sign}(\hat{n} \cdot \mathrm{C})+\left[\frac{1}{4}(1+\operatorname{sign}(n \cdot \mathrm{C})]\right.\right. \\
{\left[1+\operatorname{sign}\left\{\left(1-\frac{R^{2}}{r^{2}}\right)^{1 / 2}-\frac{\hat{n} \cdot \mathrm{C}}{C}\right\}\right] }
\end{aligned}
$$

and it has the properties that for $r$ approaching infinity, $S$ approaches 1, while for $r$ approaching the sphere $R$,

$$
S=\frac{1}{2}(1-\operatorname{sign} \hat{n} \cdot \mathbf{C}) .
$$

Furthermore, if $\theta$ is the angle between $\mathbf{r}$ and $\mathbf{C}$ then, as can be seen from Fig. 3,

$$
\begin{aligned}
& S=0 \text { if } 1 \geqslant \cos \theta \geqslant\left(1-\frac{R^{2}}{r^{2}}\right)^{1 / 2}=\cos \theta_{1} \\
& S=1 \text { if } \cos \theta<\cos \theta_{1} .
\end{aligned}
$$




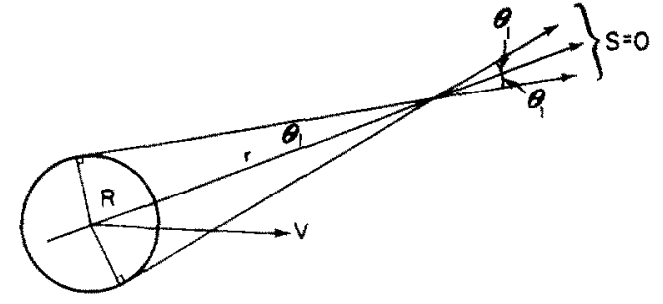

Fig. 3. Region where $S=0$.

The Density Distribution for Ions and Electrons in the Neighbourhood of the Sphere for the Case of Diffuse Reflection

The necessary calculations are considerably simplified if it is assumed that only diffuse reflection occurs so that $\alpha$ may be set equal to unity. Fortunately this appears to be a good approximation to the physical situation. ${ }^{(6,10,11)}$

We shall therefore calculate

$$
\int f(1) d^{3} \mathbf{C}
$$

and take up the two terms separately, treating first the contribution

$$
I_{1}=\frac{n}{\pi^{3 / 2}} \int S \exp -(\mathrm{C}-\mathrm{V})^{2} d V
$$

from the main stream. To evaluate $I_{1}$ we determine $S$ in rectangular $C$ space $C_{x}, C_{y}, C_{z}$. For convenience set $n$ in the direction of $C_{z}$. If

$$
\left(1-\frac{R^{2}}{r^{2}}\right)^{1 / 2}-\frac{C_{z}}{\left(C_{x}^{2}+C_{y}^{2}+C_{z}^{2}\right)^{1 / 2}}<0
$$

then $C_{z}>0$ and

$$
S=\frac{1}{2}\left[1-\operatorname{sign} C_{z}\right]=0, C_{z}>0 .
$$

Also, for all $\mathbf{C}$ for which

$$
\left(1-\frac{R^{2}}{r^{2}}\right)^{1 / 2}-\frac{C_{z}}{\left(C_{x}^{2}+C_{y}^{2}+C_{z}^{2}\right)^{1 / 2}}>0,
$$

$S=1$. Since both sides of the first inequality are positive it may be squared and solved for $C_{x}$. The result is

$$
C_{s}>\left(\frac{r^{2}}{R^{2}}-1\right)^{1 / 2}\left(C_{x}^{2}+C_{y}^{2}\right)^{1 / 2} \equiv C_{z 0}
$$

so that if $C_{z} \geqslant C_{z_{0}}, S=0$. Similarly if $C_{z}<C_{z_{0}}$, $S=1$.

Thus we must evaluate

$$
\begin{aligned}
\mathrm{I}_{1}=\frac{n}{\pi^{3 / 2}} \int_{-\infty}^{\infty} d C_{x} \int_{-\infty}^{\infty} d C_{y} \int_{-\infty}^{C_{z_{0}}} d C_{z} \exp - \\
\quad-\left[\left(C_{x}-V_{x}\right)^{2}+\left(C_{y}-V_{y}\right)^{2}+\left(C_{z}-V_{z}\right)^{2}\right] .
\end{aligned}
$$

The $C_{z}$ integral is

$$
\begin{aligned}
& \int_{-\infty}^{C_{z_{0}}} \exp -\left(C_{z}-V_{z}\right)^{2} d C_{z}=\int_{-\infty}^{C_{z_{0}}-V_{z}} \\
& \exp \left(-t^{2}\right) d t=\frac{\sqrt{ } \pi}{2}\left[\operatorname{erf}\left(C_{z_{0}}-V_{z}\right)+1\right] .
\end{aligned}
$$

Hence

$$
\begin{array}{r}
I_{1}=\frac{n}{2}+\frac{n}{2 \pi} \int_{-\infty}^{\infty} \int_{-\infty}^{\infty}-\left[\left(C_{x}-V_{x}\right)^{2}+\left(C_{y}-V_{y}\right)^{2}\right] \\
\operatorname{erf}\left(C_{z_{0}}-V\right)^{2} d C_{x} d C_{y} .
\end{array}
$$

Introduce polar co-ordinates as follows:

$$
\begin{array}{ll}
C_{x}=\rho \cos \phi & V_{x}=\sqrt{ }\left(V^{2}-V_{z}^{2}\right) \cos \psi \\
C_{y}=\rho \sin \phi & V_{y}=\sqrt{ }\left(V^{2}-V_{z}^{2}\right) \sin \psi .
\end{array}
$$

Then

$$
\begin{aligned}
& I_{1}= \frac{n}{2}\left\{1+\frac{1}{\pi} \exp -\left(V^{2}-V_{z}^{2}\right) \int_{0}^{\infty} d \rho \rho\right. \\
& \exp \left(-\rho^{2}\right) \operatorname{erf}\left[\rho /\left(\frac{r^{2}}{R^{2}}-1\right)-V_{z}\right] \times \\
&\left.\quad \times \int_{0}^{2 \pi} \exp \left[2 \rho \sqrt{ }\left(V^{2}-V_{z}^{2}\right) \cos (\phi-\psi)\right] d \phi\right\} \\
&=\frac{n}{2}\left\{1+2 \exp -\left(V^{2}-V_{z}^{2}\right) \int_{0}^{\infty} d \rho \rho \exp \left(-\rho^{2}\right)\right. \\
&\left.\operatorname{erf}\left[\rho /\left(\frac{r^{2}}{R^{2}}-1\right)-V_{z}\right] I_{0}\left[2 \rho \sqrt{ }\left(V^{2}-V_{z}^{2}\right)\right]\right\}
\end{aligned}
$$

where $I_{0}[]$ is the Bessel function of imaginary argument and zero order and

$$
\begin{aligned}
& V_{z}=\mathrm{V} \cdot n=V \cos \theta_{0} \\
& V^{2}-V_{z}^{2}=V^{2} \sin ^{2} \theta
\end{aligned}
$$


Hence

$$
\begin{aligned}
I_{1}= & \frac{n}{2}\left\{1+2 \exp \left(-V^{2} \sin ^{2} \theta_{0}\right) \int_{0}^{\infty} d \rho \rho \exp \left(-\rho^{2}\right)\right. \\
& \left.\operatorname{erf}\left[\rho /\left(\frac{r^{2}}{R^{2}}-1\right)-V \cos \theta_{0}\right] I_{0}\left(2 \rho V \sin \theta_{0}\right)\right\}
\end{aligned}
$$

In the limiting case, $r=R$,

$$
\begin{array}{r}
I_{1}=\frac{n}{2}\left\{1+2 \exp \left(-V^{2} \sin ^{2} \theta_{0}\right) \operatorname{erf}\left(-V \cos \theta_{0}\right)\right. \\
\left.\int_{0}^{\infty} d \rho \rho \exp \left(-\rho^{2}\right) I_{0}\left(2 \rho V \sin \theta_{0}\right)\right\} .
\end{array}
$$

To carry out the integration, integrate once by parts to obtain

$$
\begin{aligned}
1=\int_{0}^{\infty} \exp \left(-\rho^{2}\right) \rho I_{0}(2 u \rho)=-\left.\frac{1}{2} \exp \left(-\rho^{2}\right)\right|_{0} ^{\infty} \\
+u \int_{0}^{\infty} \exp \left(-\rho^{2}\right) I_{1}(2 u \rho) d \rho,
\end{aligned}
$$

with $u=V \sin \theta_{0}$. The integral on the right is given in the Bateman Manuscript Project series $^{(12)}$ leading to the result

$$
I=\frac{1}{2}+\frac{\sqrt{ } \pi}{2} u \exp \left(\frac{u^{2}}{2}\right) I_{1 / 2}\left(\frac{u^{2}}{2}\right) .
$$

Since

$$
I_{1 / 2}(z)=\sqrt{ }\left(\frac{2}{\pi z}\right) \sinh z
$$

one gets

$$
I=\frac{1}{2} \exp \left(u^{2}\right),
$$

and hence for $r=R$,

$I_{1}=\frac{n}{2}\left\{1+\operatorname{erf}\left(-V \cos \theta_{0}\right)\right\}=\frac{n}{2} \operatorname{erfc}\left(V \cos \theta_{0}\right)$.

This checks the direct integration of $I_{1}$ for $r$ set equal to $R$ in advance, since in this case $S=1$ only if $C_{x} \leqslant 0$.

When $r \rightarrow \infty$,

$$
\operatorname{erf}\left(\rho \sqrt{\frac{r^{2}}{R^{2}}-1}-V_{z}\right) \rightarrow \operatorname{erf}(\infty)=1
$$

and one obtains

$$
I_{1} \rightarrow \frac{n}{2}\{1+1\}=n
$$

as one should.

For the two cases of $\theta_{0}=0$ and $\theta_{0}=\pi$ the quantity $I_{1}$ can be evaluated exactly if spherical co-ordinates are used. If one uses $r$ as axis

$$
\begin{aligned}
& \mathbf{r}=r(0,0,1) \\
& \mathbf{V}=V\left(\sin \theta_{0} \cos \phi_{0}, \sin \theta_{0} \sin \phi_{0}, \cos \theta_{0}\right) \\
& \mathbf{C}=C(\sin \theta \cos \phi, \sin \theta \sin \phi, \cos \theta)
\end{aligned}
$$

and

$$
\begin{gathered}
I_{1}=\frac{n}{\pi^{3 / 2}} \int_{0}^{\infty} C^{2} d c \int_{0}^{2 \pi} d \phi \int_{0}^{\theta_{1}} \sin \theta \\
\exp \left\{-\left(C^{2}+V^{2}\right)+2 C V\left[\cos \theta \cos \theta_{0}+\right.\right. \\
\left.+\sin \theta \sin \theta_{0}\left(\cos \phi-{ }_{0}\right]\right\} d \theta .
\end{gathered}
$$

By straightforward integration of this form of $I_{1}$ when $\theta_{0}=0$ one finds

$$
\begin{aligned}
I_{1}(0)= & \frac{n}{2}\left\{\operatorname{erfc} V+\exp \left(-V^{2} \sin ^{2} \theta_{1}\right)\right. \\
& \left.\cos \theta_{1} \operatorname{erfc}\left[-V \cos \theta_{1}\right]\right\} \\
= & \frac{n}{2}\left\{\operatorname{erfc} V+\sqrt{\left(1-\frac{R^{2}}{r^{2}}\right)}\right. \\
& \left.\exp \left(-V^{2} \frac{R^{2}}{r^{2}}\right) \operatorname{erfc}\left[-V \int\left(1-\frac{R^{2}}{r^{2}}\right)\right]\right\} .
\end{aligned}
$$

A similar discussion for $\theta_{0}=\pi$ leads to the value

$$
\begin{aligned}
I_{1}(\pi)=\frac{n}{2}\left\{\operatorname{erfc}(-V)+\sqrt{\left(1-\frac{R^{2}}{r^{2}}\right)}\right. \\
\left.\quad \exp \left(-V^{2} \frac{R^{2}}{r^{2}}\right) \operatorname{erfc}\left[V /\left(1-\frac{R^{2}}{r^{2}}\right)\right]\right\} .
\end{aligned}
$$

For $r=R$ both of these reduce to our previously obtained approximation since the additional term is zero there. Likewise in the limit as $r \rightarrow \infty$ we obtain the free stream density as we should. 
For either $\theta=0$ or $\theta=\pi$ the above reduces to

$$
\lim _{r \rightarrow \infty} I_{1}=\frac{n}{2}[\operatorname{erfc}(V)+\operatorname{erfc}(-V)]=n
$$

which is correct.

To evaluate the term containing the effect of the diffusely reflected particles, it is necessary to consider the integral

$$
\begin{aligned}
& I_{2}= \frac{n}{\pi^{3 / 2}} \int d^{3} C\left[\exp -\left(\mathbf{V} \cdot \hat{n}^{\prime}\right)^{2}-\right. \\
&\left.\sqrt{ } \pi\left(\mathbf{V} \cdot \hat{n}^{\prime}\right) \operatorname{erfc}\left(\mathbf{V} \cdot \hat{n}^{\prime}\right)\right](1-S) \exp \left(-C^{2}\right) .
\end{aligned}
$$

Introducing $r$ as the polar axis again so that

$$
\begin{gathered}
\hat{n}^{\prime}=\{(-\cos \theta) r / R+r / R \\
\left.\sqrt{ }\left[\cos ^{2} \theta-\left(1-R^{2} / r^{2}\right)\right]\right\} \sin \theta \cos \phi, \\
\{(-\cos \theta) r / R+r / R \\
\left.\sqrt{ }\left[\cos ^{2} \theta-\left(1-R^{2} / r^{2}\right)\right]\right\} \sin \theta \sin \phi, \\
(r / R)\{(-r \cos \theta) / R+r / R \\
\left.\sqrt{ }\left[\cos ^{2} \theta-\left(1-R^{2} / r^{2}\right)\right]\right\} \cos \theta
\end{gathered}
$$

leads to

$$
\begin{gathered}
V \cdot \hat{n}^{\prime}=\frac{V r}{R}\left\{\cos \theta_{0}-\right. \\
{\left[\cos \theta-\sqrt{\cos ^{2} \theta-\left(1-R^{2} / r^{2}\right)}\right]} \\
\left.\left[\cos \theta \cos \theta_{0}+\sin \theta \sin \theta_{0} \cos \left(\phi-\phi_{0}\right)\right]\right\}
\end{gathered}
$$

so that $I_{2}$ is quite intractable without approximation. However, as mentioned earlier $(1-S)=1$ if and only if

$$
0 \leqslant \theta \leqslant \theta_{1}=\operatorname{arcos}\left(1-\frac{R^{2}}{r^{2}}\right)^{1 / 2}
$$

and in this range

$$
\cos \theta-\sqrt{\left[\cos ^{2} \theta-\left(1-\frac{R^{2}}{r^{2}}\right)\right]}
$$

is a very slowly varying function which has extremes at $\theta=0$ and $\theta=\theta_{1}$, with values $(1-R / r)$ and $\sqrt{ }\left(1-R^{2} / r^{2}\right)$, respectively. We will therefore replace the complicated expression for $\left(V . \hat{n}^{\prime}\right)$ in $\exp -\left(V \cdot \hat{n}^{\prime}\right)$ and erfc $\left(V \cdot \hat{n}^{\prime}\right)$ by its first term

$$
V \cdot \hat{n}^{\prime} \cong V r \frac{\cos \theta_{0}}{R} \text {. }
$$

The integral $I_{2}$ is therefore evaluated as

$$
\begin{aligned}
& I_{2} \cong I_{2}^{A}=\frac{n}{\pi^{3 / 2}} \int_{0}^{\infty} \exp \left(-C^{2}\right) C^{2} d C \int_{0}^{2 \pi} d \phi \\
& \int_{\theta=0}^{\theta_{1}} \sin \theta d \theta[ \exp \left(-\frac{V^{2} r^{2}}{R^{2}} \cos ^{2} \theta_{0}-\right. \\
&\left.\sqrt{ } \pi\left(\mathrm{V} \cdot \hat{n}^{\prime}\right) \operatorname{erfc}\left(\frac{V r \cos \theta_{0}}{R}\right)\right]
\end{aligned}
$$

where the full expression for $\left(\mathbf{V} \cdot \hat{n}^{\prime}\right)$ is used where it appears explicitly. Straightforward integration then yields the following expression for $I_{2}^{A}$

$$
\begin{aligned}
& I_{2}^{A}= \frac{n}{2}[-\cos \theta] \exp \left(-\frac{V^{2} r^{2}}{R^{2}} \cos ^{2} \theta_{0}\right)- \\
& \frac{n}{4 \pi} \int_{0}^{2 \pi} \sqrt{ } \pi \operatorname{erfc}\left(\frac{V r}{R} \cos \theta_{0}\right) \int_{0}^{\theta_{1}}\left(V \cdot \hat{n}^{\prime}\right) \sin \theta d \theta d \phi \\
&= \frac{n}{2}\left[1-\sqrt{\left.\left(1-\frac{R^{2}}{r^{2}}\right)\right] \exp \left(-\frac{V^{2} r^{2}}{R^{2}} \cos ^{2} \theta_{0}\right)-}\right. \\
& \frac{n}{2} \sqrt{ } \pi \operatorname{erfc}\left(\frac{V r}{R} \cos \theta_{0}\right) I_{3}
\end{aligned}
$$

where

$$
I_{3}=\int_{0}^{2 \pi} \int_{0}^{\theta_{1}}\left(\mathbf{V} \cdot \hat{n}^{\prime}\right) \sin \theta d \theta d \phi
$$

$$
\begin{aligned}
& =2 \pi \int_{0}^{\theta_{1}} \frac{V r}{R}\left\{\cos \theta_{0}-\left[\cos \theta-\sqrt{\cos ^{2} \theta-\left(1-\frac{R^{2}}{r^{2}}\right)}\right]\right. \\
& \left.\cos \theta \cos \theta_{0}\right\} \sin \theta d \theta \\
& =2 \pi \frac{V r}{R} \cos \theta_{0}\left\{1-\sqrt{\left(1-\frac{R^{2}}{r^{2}}\right)+}\right. \\
& \left.+\frac{1}{3} \frac{R^{3}}{r^{3}}-\frac{1}{3}\left[1-\left(1-\frac{R^{2}}{r^{2}}\right)^{3 / 2}\right]\right\} .
\end{aligned}
$$

The approximation of $I_{2}$ by $I_{2}^{A}$ is poorest in intermediate ranges of $r$ and hence it is of interest to have an upper bound in this region. The difference between $I_{2}^{A}$ and $I_{2}$ is bounded above by $\left\{\exp \left[-\left(\frac{V r}{R}\right)^{2}\left(\cos \theta_{0}+\sqrt{1-\frac{R^{2}}{r^{2}}}\right)^{2}\right]\right.$ 


$$
\begin{aligned}
& \left.-\exp \left[-\left(\frac{V r}{R}\right)^{2} \cos ^{2} \theta_{0}\right]\right\} \cdot\left(1-\sqrt{1-\frac{R^{2}}{r^{2}}}\right)+ \\
& +\sqrt{ } \pi\left(\frac{V r}{R}\right)^{2} \sqrt{1-\frac{R^{2}}{r^{2}}} \cos \theta_{0} \\
& \times \quad \exp \left[-\left(\frac{V r}{R}\right)^{2}\left(\cos \theta_{0}+\sqrt{\left.1-\frac{R^{2}}{r^{2}}\right)^{2}}\right] \times\right. \\
& \times\left\{1-\sqrt{1-\frac{R^{2}}{r^{2}}+\frac{1}{3} \frac{R^{3}}{r^{3}}-}-\frac{1}{3}\left[1-\left(1-\frac{R^{2}}{r^{2}}\right)^{3 / 2}\right]\right\}
\end{aligned}
$$

$2\left|\cos \theta_{0}\right|$. The plus sign is to be used for $\cos \theta_{0}$ $<0$ and the minus sign for $\cos \theta_{0}>0$.

\section{NUMERICAL RESULTS FOR ELECTRON DENSITY DISTRIBUTION AND RADAR CROSS-SECTIONS}

The formulas of Part 2 were applied to a typical $^{(7)}$ case of interest for which $V=5$. This corresponds, for example, to a satellite altitude of $500 \mathrm{~km}$ and speed $V^{\prime}=8 \mathrm{~km} / \mathrm{sec}$. Curves of constant relative density $N / n$ are presented in Figs. 4 and 5. They clearly show the build-up of density ahead of the sphere and the "hole" developed in the rear. The ion deficiency in the for all $r$ and $\theta_{0}$ such that $\sqrt{ }\left[1-\left(R^{2} / r^{2}\right)\right]<$ rear extends to about 50 sphere radii.

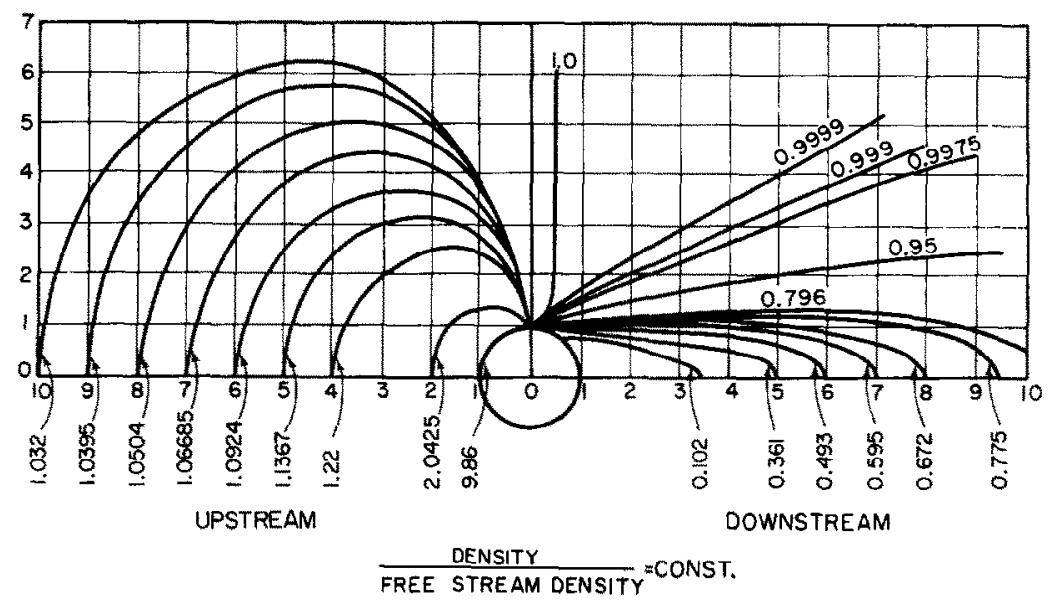

Fig. 4. Equi-density contours.

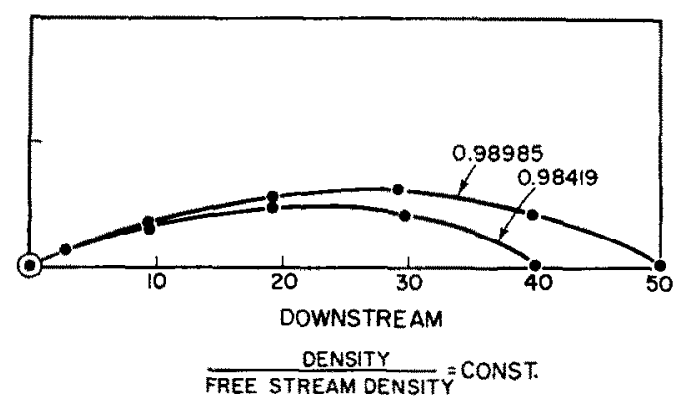

Fig. 5. Downstream equi-density contours.
More detailed data for $N$ on the sphere and along the positive $z$ axis (behind the sphere) is given in Table 1.

Radar cross-sections ( $4 \pi$ times differential cross-sections) were computed from the formula

$$
\sigma=4 \pi \sigma_{e} n^{2}\left|\int_{V}\left(\frac{N}{n}-1\right) e^{2 i k r} d v\right|^{2}
$$

according to the development in Part 1. For electrons $4 \pi \sigma_{e} \sim 10^{-28} \mathrm{~m}^{2}$. A value of $n=10^{12}$ electrons $/ \mathrm{m}^{3}$ is used for the electron density at 
$500 \mathrm{~km}$ altitude and the resulting values of $\sigma$ as well as

$$
\int\left(\frac{N}{n}-1\right) e^{2 i k r} d v
$$

are given in Table 2 for various regions $V$. It was assumed that $k=(2 \pi / \hat{\lambda})=1 \mathrm{~m}^{-1}$, that the sphere radius is $1 \mathrm{~m}$, and that the sphere was viewed broadside, i.e. $\Theta=90^{\circ}$.

Table 1. Density ratio $=$ Density/Free Stream

$$
\text { Density }=\frac{N}{n}
$$

\begin{tabular}{c|c|c|c}
\hline \multicolumn{2}{c|}{ On sphere $(r=R)$} & \multicolumn{2}{|c}{ Behind sphere (at $\left.0^{\circ}\right)$} \\
\hline$\theta$ & $N / n$ & $z$ & $N / n$ \\
\hline $0^{\circ}$ & $9.0 \times 10^{-18}$ & 1 & $9.0 \times 10^{-13}$ \\
$5^{\circ}$ & $1.0923 \times 10^{-12}$ & 2 & $1.6718 \times 10^{-3}$ \\
$10^{\circ}$ & $1.9443 \times 10^{-12}$ & 3 & $5.8621 \times 10^{-2}$ \\
$15^{\circ}$ & $4.9876 \times 10^{-12}$ & 4 & $2.0296 \times 10^{-1}$ \\
$20^{\circ}$ & $1.7947 \times 10^{-11}$ & 5 & $3.6045 \times 10^{-1}$ \\
$25^{\circ}$ & $8.7137 \times 10^{-11}$ & 6 & $4.9237 \times 10^{-1}$ \\
$30^{\circ}$ & $5.4587 \times 10^{-10}$ & 7 & $5.9422 \times 10^{-1}$ \\
$35^{\circ}$ & $4.1834 \times 10^{-8}$ & 8 & $6.7133 \times 10^{-1}$ \\
$40^{\circ}$ & $3.6932 \times 10^{-8}$ & 8.5 & $7.0258 \times 10^{-1}$ \\
$45^{\circ}$ & $3.5343 \times 10^{-7}$ & 9 & $7.2990 \times 10^{-1}$ \\
$50^{\circ}$ & $3.4398 \times 10^{-8}$ & $9 \cdot 5$ & $7.5384 \times 10^{-1}$ \\
$55^{\circ}$ & $3.1943 \times 10^{-5}$ & 10 & $7.7490 \times 10^{-1}$ \\
$60^{\circ}$ & $2.6707 \times 10^{-4}$ & 20 & $9.3824 \times 10^{-1}$ \\
$65^{\circ}$ & $1.9583 \times 10^{-3}$ & 30 & $9.7206 \times 10^{-1}$ \\
$70^{\circ}$ & $1.1018 \times 10^{-2}$ & 40 & $9.8419 \times 10^{-1}$ \\
$75^{\circ}$ & $5.0194 \times 10^{-2}$ & 50 & $9.8985 \times 10^{-1}$ \\
$80^{\circ}$ & $1.7613 \times 10^{-1}$ & & \\
$85^{\circ}$ & $4.7471 \times 10^{-1}$ & & \\
$86^{\circ}$ & $5.6145 \times 10^{-1}$ & & \\
$87^{\circ}$ & $6.5761 \times 10^{-1}$ & & \\
$88^{\circ}$ & $7.6306 \times 10^{-1}$ & & \\
$89^{\circ}$ & $8.7739 \times 10^{-1}$ & & \\
$90^{\circ}$ & 1.0 & \\
\hline \hline
\end{tabular}

Table 2

\begin{tabular}{l|c|c}
\hline \multicolumn{1}{c|}{ Region (V) } & $\frac{1}{R^{3}} \int\left(\frac{N}{n}-1\right) e^{9} i k r d v$ & $\sigma\left(\mathrm{cm}^{9}\right)$ \\
\hline$z>0$ (behind) & $-1 \cdot 1$ & $1 \cdot 3$ \\
\hline$z<0$ (ahead) & 0.64 & 0.41 \\
\hline$-\infty<z<\infty$ entire cloud & -.48 & 0.23 \\
\hline $\begin{array}{l}-\infty<z<\infty \text { entire cloud, } \\
\text { with power contribution } \\
\text { from } z<0 \text { and } z<0 \text { added } \\
\text { (i.e. the average result for } \\
\text { random relative phase) }\end{array}$ & & 1.7 \\
\hline \hline
\end{tabular}

The first two $\sigma$ values above are of interest if the radar beam is respectively lagging or leading the satellite while the $1.7 \mathrm{~cm}^{2}$ result would be most appropriate, for example, if many radar pulses are being received and added with a radar which is tracking inaccurately, "hunting" around the target.

It is of interest to compare these results with a comparable cross-section estimate obtained by Davis ${ }^{(13)}$ in a much cruder but far simpler fashion. Davis neglected the density build-up ahead of the sphere and assumed the electrons were completely swept out of a cylindrical column one sphere diameter wide. On this assumption his computations led to a length estimate of 10 sphere diameters behind the sphere. The radar return from such a cavity is that of a column of electrons embedded in a vacuum and of electron number density given by the unperturbed number density. This number of electrons per unit volume was then referred to an equivalent line density and the problem replaced by that of coherent scattering by a line source for which the cross-section is proportional to line density times $\lambda^{4}$. To scale Davis's numerical result of $\sigma=0.1 \mathrm{~cm}^{2}$ for a $0.25 \mathrm{~m}$ radius sphere in a medium of $n=10^{12} / \mathrm{m}^{2}$ to the present $1 \mathrm{~m}$ radius sphere problem his equivalent line density $\left(2 \times 10^{9} / \mathrm{cm}\right)$ is scaled by $(100 / 25)^{2}=16$ and the fact that $\sigma$ is proportional to line density squared leads to a scale factor of 256 . On the other hand the present computations were made for $\lambda=2 \pi \mathrm{m}$ while Davis apparently used $15 \mathrm{~m}$. Hence an additional wavelength scaling factor of $(2 \pi / 15)^{4}$ is needed or a net scale factor of $\sim 8$. The crosssection value to compare with ours is thus

$$
\sigma_{D} \sim 0.8 \mathrm{~cm}^{2} \text {. }
$$

An instructive insight into the behaviour of the perturbation at various distances ahead or behind the sphere is furnished by a plot of the contribution to the volume integral of the various axial stations; i.e. a plot of

$$
S(z)=\int_{0}^{\infty} \rho d \rho\left(\frac{N}{n}-1\right) J_{0}[2 k \rho \sin \theta]
$$


vs. $z$. This is furnished in Fig. 6. The somewhat odd transition in $S(z)$ as $z$ crosses the origin reflects the fact that $N$ within the sphere is zero. To complete the volume integration this function is to be multiplied by $\exp \left[2 i k\left(\frac{z^{2}}{2 R}-z \cos \theta\right)\right]$ and integrated. It is clear that as $\Theta$ and hence $\theta$ deviate from $90^{\circ}$ or as $k$ increases the rate of oscillation of the exponential will increase and the net contributions from both regions $z>0$ and $z<0$, will rapidly decrease

Acknowledgements-The authors would like to thank Professor K. M. Siegel for suggesting the physical bases which enabled the use of the postulate of a neutral gas to be used for determining the ion and electron distributions. The authors would also like to acknowledge helpful discussions with Professor G. E. Uhlenbeck and Professor P. C. Clemmow. Finally, the authors wish to acknowledge the help of Larry $\mathbf{B}$. Evans in coding the formulas for computation and to express their appreciation to The University of Michigan Computing Centre, directed by Professor R. C. F. Bartels, for the computing time necessary to complete this work.

\section{REFERENCES}

1. H. BRYSK, J. Geophys. Res. 63, 693 (1958).

2. L. Kraus and K. M. Watson, Phys, of Fluids 1, 40 (1958).

3. P. Greifinger, RAND Rep. R339 No. 19 (June 1959).

4. R. PAPPERT, Convair Rep. ZPH-026 (Dec. 1958).

5. D. PINes and R. Bohm, Phys. Rev. 85, 338 (1952).

6. C. S. Wang Chang, Univ. Michigan Eng. Res. Inst. Rep. CM-654 (Dec. 1950).

7. L. SPITZER, Jr., Physics of Fully Ionized Gases. Interscience, New York (1956).

8. C. L. DolPH and R. K. OsBorn, Univ. Michigan Radiation Lab. Memo No. 2764-547-M (1959).

9. I. B. BERnSTEIN and I. N. RABINowITZ, Physics of Fluids 2, 112 (1959).

10. U. R. Stalder and V. J. ZURICK, NACA TN 2423 (July 1951).

11. F. C. Hurlbut, RAND Rep. R339 No. 21 (June 1959).

12. A. ERDelyl editor, Higher Transcendental Functions, Vol. 2, Eq. 27, p. 92. McGraw-Hill, New York (1953).

13. A. DAvis, Proceedings of the Conference on Interaction of Satellites with the Ionosphere, pp. 33.34, U.S. Naval Research Lab., Washington, D.C. (March 1958).

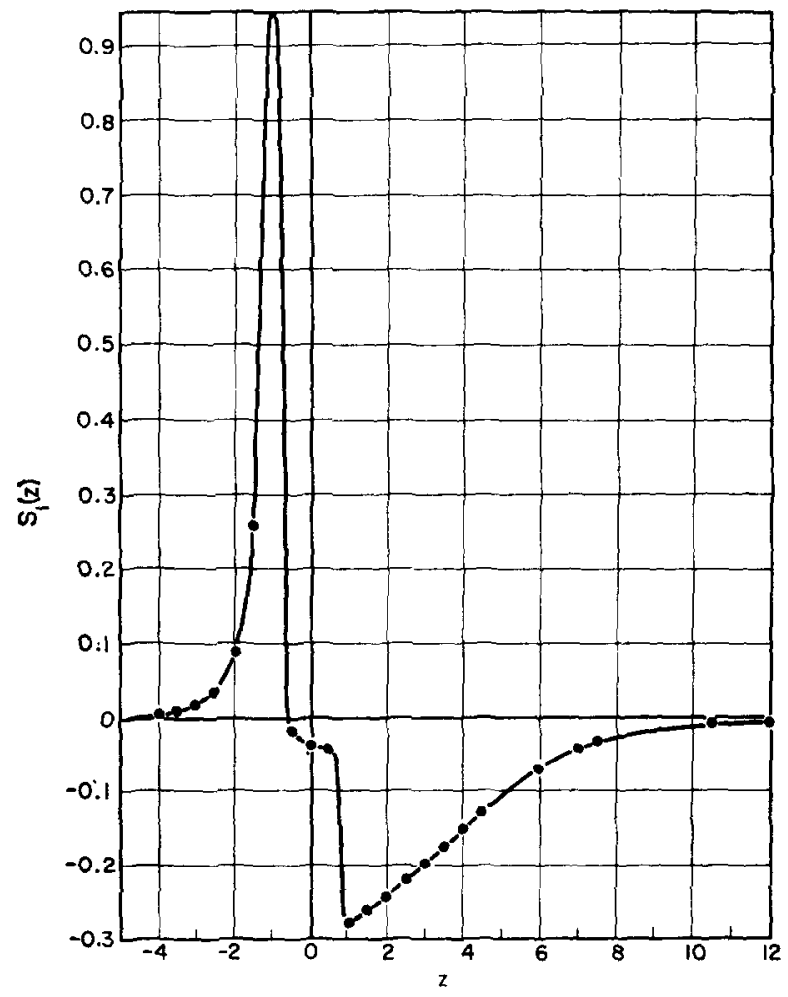

Fig. 6. Contribution to cross-section of the axial locations. 